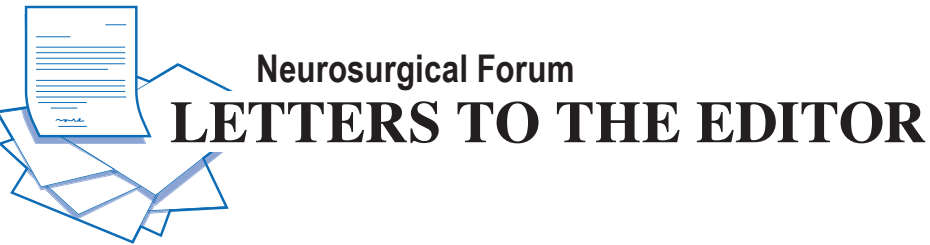

\section{Clipping after Pipeline embolization device placement for a thrombosed ACoA aneurysm}

TO THE EDITOR: We read with great interest the article published by Daou and colleagues ${ }^{2}$ (Daou B, Chalouhi N, Starke RM, et al: Clipping of previously coiled cerebral aneurysms: efficacy, safety, and predictors in a cohort of 111 patients. J Neurosurg 125:1337-1343, December 2016). This publication has enormous interest for cerebrovascular neurosurgeons working in centers that offer both endovascular and surgical aneurysm repair strategies. Surgical treatment of aneurysms previously treated with endovascular therapy presents several constraints, as mentioned in this article. We would like to provide from our practice an additional example of clipping an aneurysm that had been previously treated with a stent as we think it would be interesting to readers of the cited article. While ruling out causes of chronic headache, we diagnosed a 70-year-old woman with an aneurysm of the anterior communicating artery (ACoA), with its neck at the left $\mathrm{A}_{1}-\mathrm{A}_{2}$ junction. The aneurysm measured $8 \times 8.5 \mathrm{~mm}$. The dome of the aneurysm was pointing anteroinferiorly and had an intraluminal thrombus (Fig. 1). She had no visual or other neurological deficits associated with the aneurysm. Considering the size and location of the aneurysm, our multidisciplinary team proposed treatment. Both surgical and endovascular options were suggested, and the patient elected to undergo the endovascular option. A $2.75 \times 16-\mathrm{mm}$ Pipeline embolization device (PED) was positioned on the left $A_{1}-A_{2}$ segments under biplane fluoroscopic guidance. The immediate postprocedural digital subtraction angiography (DSA) study showed marked contrast stasis within the sac, compatible with reduced inflow. Treatment with aspirin and clopidogrel had been instituted 1 week before the procedure, without any antiplatelet resistance on biological tests. Magnetic resonance angiography (MRA) performed 1 month after treatment showed no evidence of blood flow inside the aneurysm sac, and the patient had a normal neurological examination. Three months after intervention, the patient experienced progressive diminution of vision in the left eye, and in the space of a week she had no light perception in this eye. Visual field and optical coherence tomography (OCT) findings suggested a lesion of the retrobulbar, prechiasmatic optic nerve on the left side (Fig. 2A and C). Magnetic resonance imaging, MRA, and DSA showed complete exclusion of the treated aneurysm and normal flow within the parent vessel but also a paradoxical increase in size related to intrasacular thrombosis, leading to worsening of the mass effect and optic neuropathy (Fig. 3). After discussion in the multidisciplinary neurovascular board, we elected to treat this aneurysm surgically. The optic nerve was markedly compressed by a turgid thrombosed aneurysm with solid and liquid components. The thrombosed aneurysm sac was excised after decompression of the sac contents, and the aneurysm neck was clipped. The aneuysm sac was completely dissected away from the optic nerve, thus achieving good decompression of the nerve (Fig. 4). The patient recovered her vision completely in the first 2 postoperative days, which was later confirmed with formal assesments of visual acuity, fields, and OCT (Fig. 2B and D).

As Colby and colleagues recently showed in a nonrandomized retrospective series, ${ }^{1}$ flow diversion using a PED for the repair of an ACoA aneurysm seems to have an extremely high rate of success $(96 \%)$ immediately after the procedure. With respect to long-term results, this study showed complete occlusion of the aneurysm in $86 \%$ at an average follow-up of 10.4 months. The safety and efficacy of PED placement in terms of ophthalmological outcome after coiling large and giant internal carotid artery aneurysms were evaluated by Sahlein et al. ${ }^{4}$ while analyzing the results of the Pipeline for Uncoilable or Failed Aneurysms (PUFS) trial. These authors found that the procedure was related to a $5 \%$ worsening of previous ophthalmological deficit, attributed to ischemia of the ophthalmic artery territory. Gressot et al. ${ }^{3}$ have recently shown that
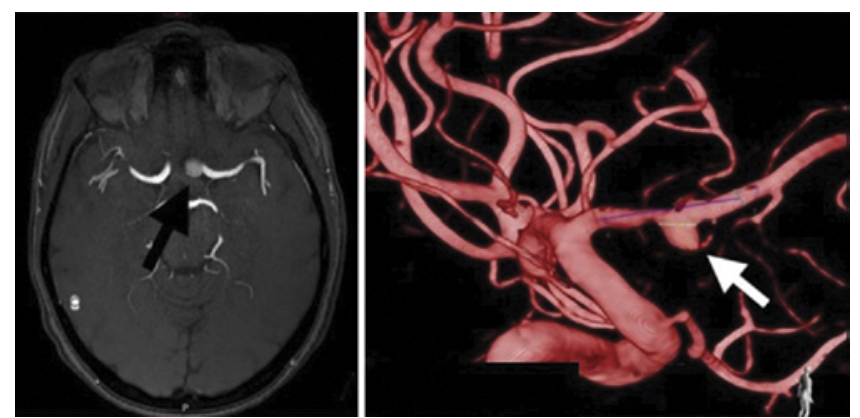

FIG. 1. Left: Initial MR angiogram showing an $8 \times 8.5-\mathrm{mm} A C o A$ aneurysm (black arrow). Right: Three-dimensional reconstruction of DS angiogram showing the circulating part of the aneurysm (white arrow). Figure is available in color online only. 


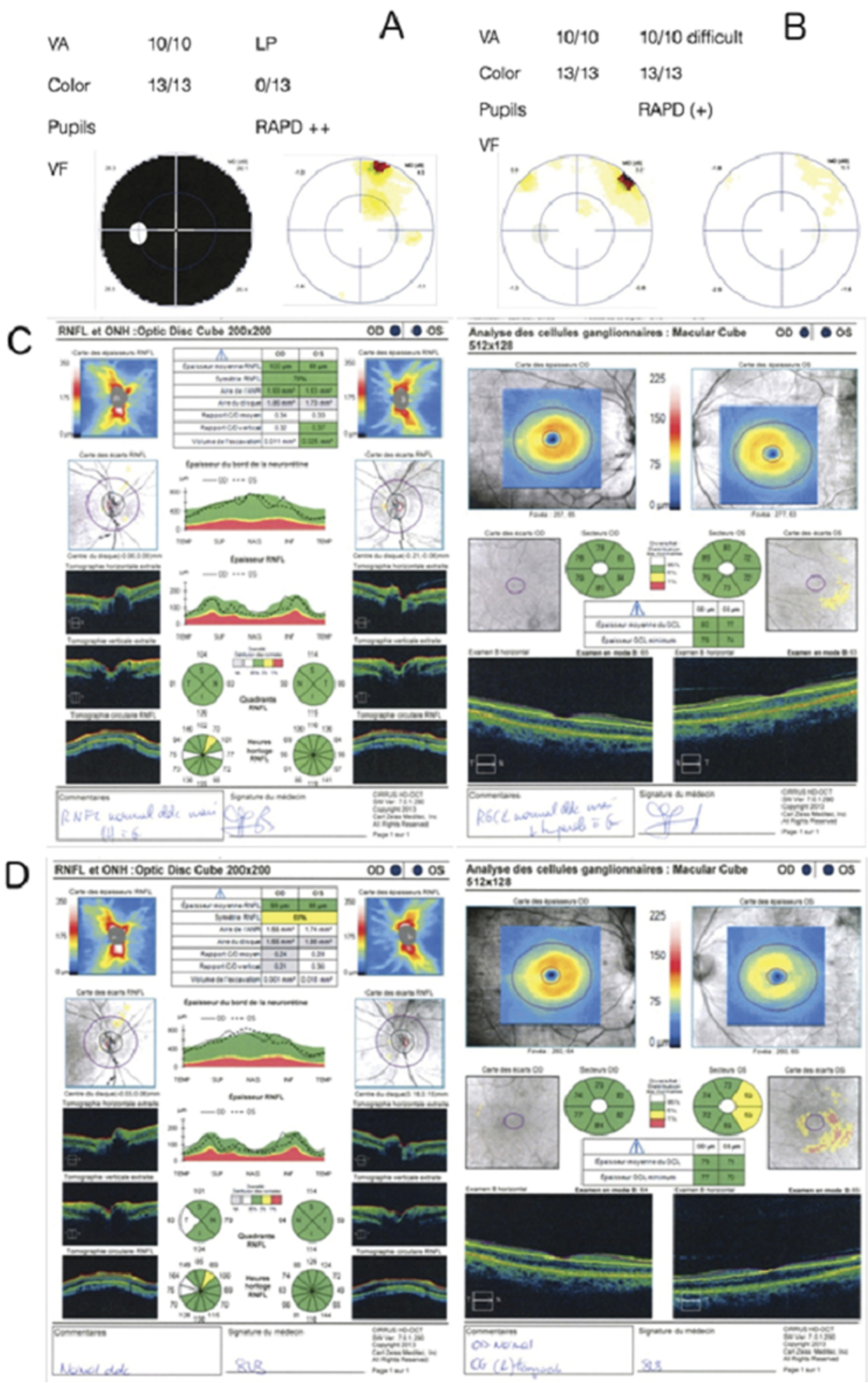

FIG. 2. A: Preoperative visual fields showing marked vision loss in the left eye. B: Postoperative visual fields showing the normalization of vision. C: Preoperative OCT showing left optic nerve impairment. D: Postoperative OCT showing the normalization of vision. Figure is available in color online only. 


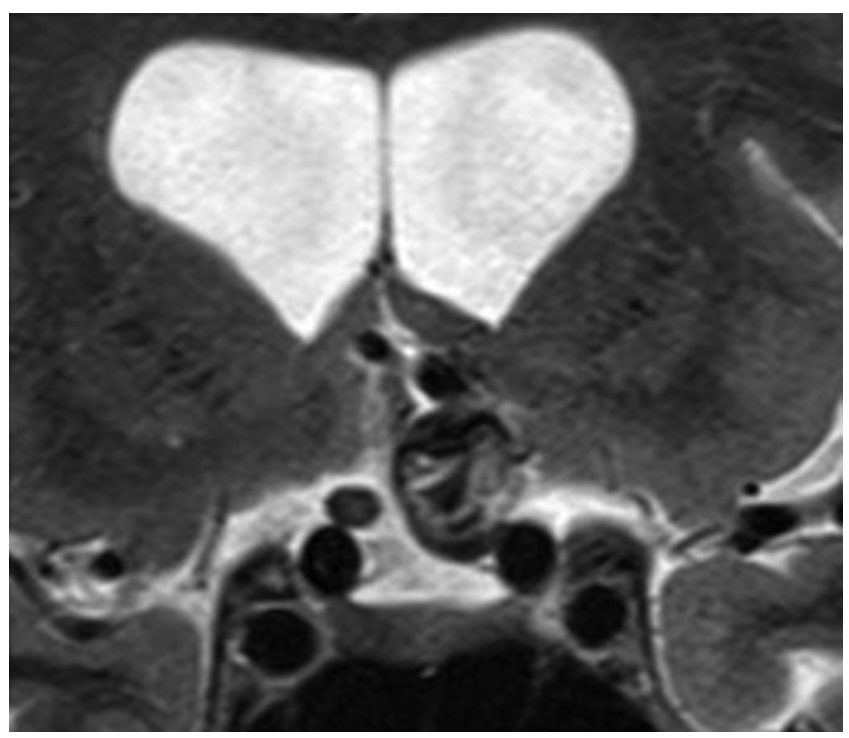

FIG. 3. Coronal T2-weighted MR image obtained 3 months after stenting, showing compression of the optic nerve by the thrombosed aneurysm dome.

certain aneurysms continue to show filling after PED placement, even though the ideal timeframe for proposing other alternatives in this situation is under debate. These authors also observed that some aneurysms may never close even after discontinuation of the usual 3 months of dual antiplatelet therapy. Ophthalmic artery aneurysms seem to be particularly refractory to treatment with PED placement, especially when the ophthalmic artery arises from the aneurysm sac. The rate of failure increases if this type of aneurysm presents as large, with a complicated morphology, or after previous endovascular treatment. ${ }^{3}$

The case that we describe is an unusual complication of a new neurological deficit that appeared tardively in a patient who had apparently successful endovascular treatment. Surgery resulted in a complete reversal of the neurological problem. The presence of a stent within the parent vessel across the aneurysm neck rendered the artery rigid and thus made clipping of the neck more difficult. To the best of our knowledge, this is the first report of a throm-
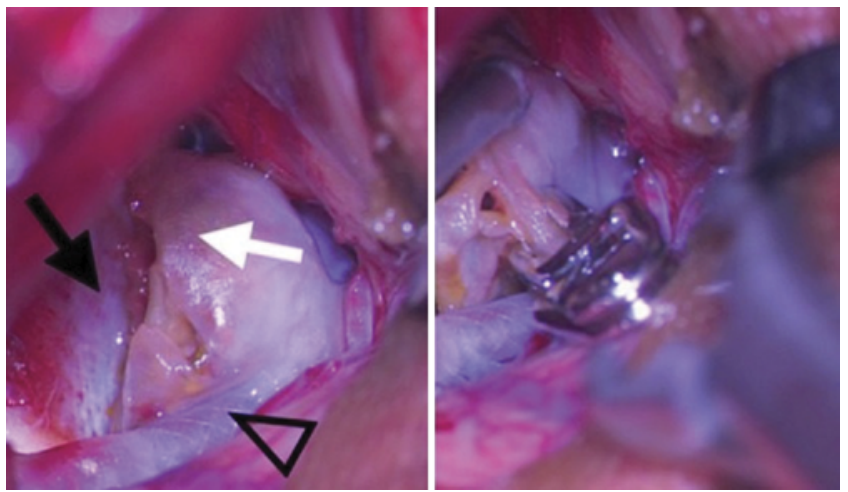

FIG. 4. Left: Intraoperative photograph showing the partially excised aneurysm (white arrow), the compressed optic nerve (black arrow), and the PED in place (black arrowhead). Right: Clipping of the aneurysm neck. Figure is available in color online only. bosed enlarging aneurysm in a patient presenting with a new, progressive neurological deficit after PED treatment. This case illustrates the need to be judicious when proposing PED treatment for freshly thrombosed aneurysms exerting mass effect on critical structures such as the optic nerve.

Mattia Pacetti, MD

Pascal J. Mosimann, MD Jean-Baptiste Zerlauth, MD

Francesco Puccinelli, MD, PhD Marc Levivier, MD, PhD

Roy Thomas Daniel, MBBS, MCh

University Hospital of Lausanne, Switzerland

\section{References}

1. Colby GP, Bender MT, Lin LM, Beaty N, Huang J, Tamargo $\mathrm{R}$, et al: Endovascular flow diversion for treatment of anterior communicating artery region cerebral aneurysms: a singlecenter cohort of 50 cases. J Neurointerv Surg [epub ahead of print], 2017

2. Daou B, Chalouhi N, Starke RM, Barros G, Ya'qoub L, Do J, et al: Clipping of previously coiled cerebral aneurysms: efficacy, safety, and predictors in a cohort of 111 patients. J Neurosurg 125:1337-1343, 2016

3. Gressot LV, Patel AJ, Srinivasan VM, Arthur A, Kan P, Duckworth EA: An intraoperative look at failure of flow diversion: when additional or alternative treatments should be considered. World Neurosurg 93:486.e7-486.e12, 2016

4. Sahlein DH, Fouladvand M, Becske T, Saatci I, McDougall CG, Szikora I, et al: Neuroophthalmological outcomes associated with use of the Pipeline Embolization Device: analysis of the PUFS trial results. J Neurosurg 123:897-905, 2015

\section{Disclosures}

The authors report no conflict of interest.

\section{Response}

We thank the authors for their interest in our study. They describe an interesting, well-illustrated case of a patient with a partially thrombosed ACoA aneurysm that was initially successfully treated with flow diversion resulting in complete exclusion of the aneurysm from the circulation. However, this patient suffered from optic neuropathy secondary to mass effect on the optic nerve from intrasaccular thrombosis. The authors were able to elegantly manage this complication through a craniotomy for decompression of the intrasaccular contents and clipping, with a reportedly excellent clinical outcome.

We commend the authors on their efforts in managing a very challenging case and the good outcome. At our institution, flow diversion has become the primary treatment modality for several aneurysm locations. ${ }^{1-4}$ However, we generally do not employ flow diversion for ACoA aneurysms, although, as the authors mentioned in their letter, the technique may have been associated with good results elsewhere.

It is noteworthy that the patient had complete reversal of her visual deficits in the first 2 days following decompression of the optic nerve. The natural history of intraaneurysmal thrombi developing or worsening after flow diversion remains unknown. Although we do agree with the authors' 
approach in managing this complication, it is also totally possible that thrombi spontaneously regress in the long run with the subsequent reversal of neuropathy. Our group has published a report on intraaneurysmal thrombus modification after flow diversion. ${ }^{5}$ In our experience, most patients with preexisting intraaneurysmal thrombi will have regression of the thrombi within a year after flow diversion. Symptom improvement reflects thrombus regression.

Finally, we agree with the authors that surgical clipping can be challenging after flow diversion due to rigidity of the parent vessel where the flow diverter is deployed, thus complicating the ability to obtain proximal control. In addition, surgery may have to be performed while on an antiplatelet agent to prevent the risk of a major perioperative stroke. Although patients undergoing flow diversion should be selected carefully, in our experience those with intraaneurysmal thrombi are generally good candidates for flow diversion with subsequent regression of thrombi and neurological deficits in most cases. ${ }^{5}$

Pascal Jabbour, MD Elias Atallah, MD

Nohra Chalouhi, MD

Thomas Jefferson University and Jefferson Hospital for Neuroscience, Philadelphia, PA

\section{References}

1. Chalouhi N, Chitale R, Starke RM, Jabbour P, Tjoumakaris $S$, Dumont AS, et al: Treatment of recurrent intracranial aneurysms with the Pipeline Embolization Device. J Neurointerv Surg 6:19-23, 2014

2. Chalouhi N, Jabbour P, Starke RM, Zanaty M, Tjoumakaris $\mathrm{S}$, Rosenwasser RH, et al: Treatment of a basilar trunk perforator aneurysm with the Pipeline Embolization Device: case report. Neurosurgery 74:E697-E701, 2014

3. Chalouhi N, Starke RM, Yang S, Bovenzi CD, Tjoumakaris $\mathrm{S}$, Hasan D, et al: Extending the indications of flow diversion to small, unruptured, saccular aneurysms of the anterior circulation. Stroke 45:54-58, 2014

4. Zanaty M, Chalouhi N, Tjoumakaris SI, Gonzalez LF, Rosenwasser R, Jabbour P: Flow diversion for complex middle cerebral artery aneurysms. Neuroradiology 56:381-387, 2014

5. Zanaty M, Jabbour PM, Bou Sader R, Chalouhi N, Tjoumakaris S, Rosenwasser RH, et al: Intra-aneurysmal thrombus modification after flow-diversion. J Clin Neurosci 22:105110,2015

INCLUDE WHEN CITING

Published online August 11, 2017; DOI: 10.3171/2017.3.JNS17574.

CAANS, 2017

\section{da Vinci robot-assisted transoral surgery for sellar tumors}

TO THE EDITOR: Chauvet et al. ${ }^{1}$ should be commended for completing the first clinical study on the feasibility of da Vinci robot-assisted transoral surgery for sellar tumors (Chauvet D, Hans S, Missistrano A, et al: Transoral robotic surgery for sellar tumors: first clinical study. $J$ Neurosurg [epub ahead of print December 23, 2016; DOI: 10.3171/2016.9.JNS161638]). Master-slave robotic platforms such as the da Vinci system may offer several advantages over conventional endoscopic surgery including high-resolution stereoscopic images of the operative field and articulated EndoWrists that increase surgical dexterity. Moreover, such platforms have already been used in a wide range of procedures and surgical specialties such as urology. However, we caution against the adoption of da Vinci robot-assisted transoral surgery for sellar tumors.

In its present form, lamentably, the da Vinci system is ill suited to brain surgery. A previous cadaver study revealed that its multiple large and bulky arms, limited range of instruments, and lack of haptic feedback were not compatible with the very delicate and precise maneuvers required when performing keyhole transcranial endoscopic procedures. ${ }^{2}$ Chauvet et al. themselves note that although the robot was used to create the mucosal flap, actual drilling of the sphenoid using a Midas Rex Legend Stylus (Medtronic) and removing the tumor using curettes were performed by hand at the patient's side. In addition to the aforementioned technical challenges, several other barriers exist including the very high cost of purchasing and maintaining such systems.

Chauvet et al. uniquely demonstrate the utility of the transoral approach for accessing the pituitary fossa, which is not a conventional indication for this approach but has advantages, as they mention, and does allow for the use of the da Vinci robot. Whether the addition of the da Vinci robot itself improves the surgical technique or the clinical outcome remains to be demonstrated.

Surgical robotics is a young field, and in the near future it is likely that smaller and more affordable specialty- and procedure-specific robots will replace the large and expensive multipurpose robots of today. The introduction of these new devices into the clinical arena should occur in a manner that ensures their safety and effectiveness. To this end, prospective development studies, protocol and study registries, and the development of reporting standards and definitions of key outcomes will all be important in ensuring innovation and evaluation proceed in parallel. ${ }^{3}$

\section{Hani J. Marcus, $\mathrm{PhD}^{1,2}$ David Choi, $\mathrm{PhD}^{2}$ \\ Neil L. Dorward, MS ${ }^{2}$}

${ }^{1}$ The Hamlyn Centre, Institute of Global Health Innovation, Imperial College London, London, United Kingdom ${ }^{2}$ National Hospital for Neurology and Neurosurgery, University College London Hospitals NHS Foundation Trust, London, United Kingdom

\section{References}

1. Chauvet D, Hans S, Missistrano A, Rebours C, El Bakkouri W, Lot G: Transoral robotic surgery for sellar tumors: first clinical study. J Neurosurg [epub ahead of print December 23, 2016. DOI: $10.3171 / 2016.9 . J N S 161638]$

2. Marcus HJ, Hughes-Hallett A, Cundy TP, Yang GZ, Darzi A, Nandi D: da Vinci robot-assisted keyhole neurosurgery: a cadaver study on feasibility and safety. Neurosurg Rev 38:367-371, 2015 
3. McCulloch P, Cook JA, Altman DG, Heneghan C, Diener MK: IDEAL framework for surgical innovation 1: the idea and development stages. BMJ 346:f3012, 2013

\section{Disclosures}

The authors report no conflict of interest.

\section{Response}

We thank Marcus and associates for their congratulations and their judicious remarks. It is now recognized that da Vinci robot-assisted surgery has modified some surgical specialties with lower morbidity for the patients. However, the neurosurgical field has not yet benefited from this technology for two reasons: brain surgery raises specific anatomical concerns with regard to narrow spaces, and actual master-slave robots have not been designed for microsurgery. In cadaveric studies, researchers using the da Vinci robot have attempted to perform standard keyhole neurosurgery with major limitations ${ }^{1,2}$ It is true that the da Vinci system in its present form is not perfectly suited for these transcranial approaches. But we emphasize that our study was focused on transoral robotic surgery (TORS) in the skull base. Previous otolaryngological studies have shown that the da Vinci robot serves as a valuable tool in the oral cavity, which presents with a sufficient workspace as opposed to the aforementioned cranial keyhole approaches.

More generally, the future of surgery will include robots of different kinds and/or guidance systems, especially for neurosurgery. We must acknowledge the limitations of existing as well as next-generation robotic systems. Patient safety and surgical effectiveness must remain our primary end points. However, in terms of surgical development, every surgeon should be involved in thinking about new tools that could challenge the current gold standards. All innovative work can be discussed-fortunately-and pioneers of transnasal transsphenoidal endoscopy probably remember the critiques they have sustained. With our robotic approach, we believe that jumping on the bandwagon is promising, even necessary.

Finally, beyond our presented article on using the da Vinci system, we present a new hypothesis - that the transoral approach could be an interesting means of reaching the skull base. Indeed, we emphasize two points: it prevents rhinological inconveniences and it enables a more direct approach along a new inferosuperior direction, which corresponds to the axis of pituitary adenoma growth. At this time, we have begun additional work to perform TORS with other devices.

People put limitations on their creativity, believing they have to rely on what they know and what they have done.

Bertrand Piccard (Solar Impulse cofounder)

Dorian Chauvet, MD Alister Rogers, MD Guillaume Lot, MD, PhD Fondation Ophtalmologique Rothschild, Paris, France Stéphane Hans, MD, PhD Hôpital Européen Georges Pompidou, Paris, France

\section{References}

1. Hong WC, Tsai JC, Chang SD, Sorger JM: Robotic skull base surgery via supraorbital keyhole approach: a cadaveric study. Neurosurgery 72 (Suppl 1):33-38, 2013

2. Marcus HJ, Hughes-Hallett A, Cundy TP, Yang GZ, Darzi A, Nandi D: da Vinci robot-assisted keyhole neurosurgery: a cadaver study on feasibility and safety. Neurosurg Rev 38:367-371, 2015

INCLUDE WHEN CITING

Published online June 23, 2017; DOI: 10.3171/2017.1.JNS163268.

cAANS, 2017

\section{Expression of tissue (pro)renin receptor and concentrations of its soluble form in CSF in adult diffuse gliomas}

TO THE EDITOR: We read with great interest the article by Kouchi et al. ${ }^{1}$ (Kouchi M, Shibayama Y, Ogawa D, et al: (Pro)renin receptor is crucial for glioma development via the $\mathrm{Wnt} / \beta$-catenin signaling pathway. $J$ Neurosurg [epub ahead of print January 6, 2017; DOI: 10.3171/2016.9.JNS16431]) concerning (pro)renin receptor (PRR) expression in gliomas and its role in glioma development. In their exciting work, the authors retrospectively analyzed PRR, IDHI immunoexpression, and Ki-67 labeling separately in paraffin sections of 31 gliomas. They also evaluated expression of the PRR and Wnt/ $\beta$-catenin pathway components in cultured human glioma cell lines (obtained from the American Type Culture Collection) using Western blotting. The effects of PRR short interfering RNA (siRNA) on glioma cell proliferation (WST-1 assay and direct cell counting) and apoptosis (flow cytometry and the caspase-3 assay) were also examined.

The authors reported that PRR expression was significantly higher in glioblastomas (WHO Grade IV) than in normal tissue or in lower grade gliomas (WHO Grade II or III), regardless of IDHI mutation status, and that PRR expression showed a significant negative correlation with the survival time of patients with gliomas. In vitro treatment of cell lines derived from human glioblastomas with PRR siRNA significantly reduced expression of Wnt2, activated $\beta$-catenin, and cyclin D1; reduced the proliferative capacity of these cell lines; and induced apoptosis. Therefore, the authors suggested an important role of PRR in the development of glioma by aberrant activation of the Wnt/ $\beta$-catenin signaling pathway.

For diffuse gliomas in adults, there is no established marker of long-term treatment efficiency and no generally accepted prognostic marker or biological marker of tumor progression. Thus, we really appreciate the authors' contribution in the study of a potential prognostic marker and therapeutic target.

Our team has studied PRR in glioma patients since 2012. We prospectively evaluated PRR expression in glio- 
mas $(n=14)$ and in controls $(n=16)$ using immunohistochemical (IHC) analysis (monoclonal mice anti-PRR 8A12 clone antibodies, provided by G. Nguyen) and by in situ hybridization (ISH; based on human PRR anti-sens mRNA). The concentrations of the soluble form of PRR (sPRR) in CSF in patients with gliomas $(n=14)$, nonglial tumors ( $n$ $=19)$, and in controls $(n=16)$ were also measured using an enzyme-linked immunosorbent assay (ELISA) test (Immuno-Biological Laboratories).

To the best of our knowledge, our study was the first to analyze PRR protein by IHC analysis and PRR mRNA by ISH in human glioma samples ${ }^{2}$ and one of the first to study PRR in tumor pathology in humans. ${ }^{3}$ We have also shown and measured the sPRR in the CSF of glioma patients and controls. Because of this highly variable expression, our study does not support PRR as a promising specific biomarker of infiltrative glioma cells. There was not a statistically significant difference in sPRR concentrations between glioma patients and controls $(\mathrm{p}=0.400)$, or between glioblastomas and WHO Grade II gliomas ( $\mathrm{p}$ $=0.950$ ). Epilepsy did not seem to impact sPRR levels, but sPRR levels had a tendency to be higher in older patients $(>60$ years, $\mathrm{p}=0.070)$.

Kouchi et al. demonstrated that PRR expression was significantly higher in glioblastomas than in normal tissue or in lower grade gliomas, regardless of IDHI mutation. Due to the high variation of PRR staining by IHC, even between different glioblastoma cases in our series, our results failed to confirm the higher expression of PRR in glioblastomas compared with normal tissue. It could be proposed that the PPR score mainly reflects the increase in tumoral astrocytic cell density rather than glioma grade. The authors also showed that PRR expression had a significant positive correlation with the $\mathrm{Ki}-67$ labeling index. Taking into account these results and our data regarding PRR expression in GFAP-positive cells, double immunostaining techniques and staining coupled with ISH will help to identify the sites of preferential PRR synthesis in gliomas. This work could potentially be a crucial step in research of therapeutic target for glioma.

Kouchi et al. reported that PRR IHC expression had a significant negative correlation with the survival time of glioma patients. Unfortunately, the survival data are not detailed for each glioma type and grade, and no information regarding treatment modalities is available. As the study spanned over a long period of time (2007-2015), oncological treatment of the patients could have differed with time, having an impact on overall survival. In addition, the authors' cohort size (28 glioma patients and 3 normaltissue controls $)^{1}$ and that in our series (14 gliomas patients and 16 controls) ${ }^{2}$ seem insufficient to establish PRR as a prognostic marker.

Prospective studies (ideally multicenter with unique standardized protocol), the use of recent WHO brain tumor classification for glioma diagnosis, listing of modern intra- and postoperative treatment modalities used, and accurate follow-up are needed to assess the role of PRR as a potential prognostic marker and a potential marker of tumor recurrence.
Vladislav Pavlov, MD, MSc ${ }^{1}$ Pascale Varlet, MD, $\mathrm{PhD}^{1-3}$
Fabrice Chretien, MD, PhD ${ }^{1}$ Geneviève Nguyen, $\mathrm{MD}, \mathrm{PhD}^{4}$ Johan Pallud, MD, $\mathrm{PhD}^{1-3}$

Sainte-Anne Hospital, Paris, France ${ }^{2}$ Paris Descartes University, Sorbonne Paris Cité, Paris, France ${ }^{3}$ IMA-BRAIN, INSERM U894, Centre de Psychiatrie et Neurosciences, Paris, France

${ }^{4}$ Collège de France, Center for Interdisciplinary Research in Biology, INSERM U1050, Paris, France

\section{Acknowledgments}

These collaborators are greatly acknowledged (in alphabetical order): Damien Bergerot, Bertrand Devaux, Edouard Dezamis, Takuo Hirose, and François-Xavier Roux.

\section{References}

1. Kouchi M, Shibayama Y, Ogawa D, Miyake K, Nishiyama A, Tamiya T: (Pro)renin receptor is crucial for glioma development via the $\mathrm{Wnt} / \beta$-catenin signaling pathway. $\mathbf{J}$ Neurosurg [epub ahead of print January 6, 2017. DOI: 10.3171/2016.9.JNS16431]

2. Pavlov V, Pallud J, Hirose T, Roux FX, Chrétien F, Devaux $\mathrm{B}$, et al: Expression du récepteur de la (pro)rénine dans les gliomes infiltrants de l'adulte et de sa forme soluble dans le LCR. Neurochirurgie 59:253, 2013

3. Yamamoto H, Kaneko K, Ohba K, Morimoto R, Hirose T, Satoh F, et al: Increased expression of (pro)renin receptor in aldosterone-producing adenomas. Peptides 49:68-73, 2013

\section{Disclosures}

The authors report no conflict of interest.

\section{Response}

We greatly appreciated the insightful comments by Pavlov et al. In this reply, we suggest possible reasons for the discrepancy between our findings and those of Pavlov's group. We also provide more detailed information regarding the treatment of our glioma patients.

Pavlov et al. measured sPRR in the CSF of glioma patients and controls. Because of the marked variation of the sPRR concentration among samples, there was no significant difference between glioma patients and controls or between glioblastoma and WHO Grade II glioma. In a preliminary study, we also measured the plasma and CSF levels of sPRR in glioma patients using the same commercial ELISA kit (Immuno-Biological Laboratories). We found marked variation of sPRR as reported by Pavlov et al. (data not shown), and thus we concluded that measurement of sPRR in plasma or CSF was not useful in glioma patients. Why does the relationship between intratumoral PRR expression and the sPRR concentration in plasma or CSF vary in patients with glioma? In this regard, previous studies have indicated that full-length PRR is cleaved by activation of proteases such as furin to generate sPRR, which is then released into the CSF or plasma. ${ }^{2}$ During progression of glioma, activity of the relevant proteases may be modulated by several factors. Therefore, it can be speculated that unstable activity of these proteases influences the relationship between intratumoral expression of 
PRR and plasma or CSF concentrations of SPRR in glioma patients.

Pavlov et al. suggested that the PRR score mainly reflects an increase of astrocytic cell density rather than glioma grade. However, an increase in tumoral astrocytic cell density is one of the WHO criteria for progression, ${ }^{1}$ so astrocytic cell density is strongly associated with glioma grade. We found a significant positive correlation between PRR expression and the Ki-67 labeling index. We also demonstrated that PRR expression directly regulates cell proliferation via the $\mathrm{Wnt} / \beta$-catenin signaling pathway. These 2 findings strongly suggest that PRR is substantially involved in the progression of glioma and can be used as an indicator of malignancy. As pointed out by Pavlov et al., identifying the site of preferential PRR synthesis in GFAP-positive cells by double immunostaining and staining coupled with ISH will be helpful for detailed research into therapeutic targets for glioma.

Pavlov et al. also mentioned deficiencies in our survival data for the glioma patients; thus, we take this opportunity to provide information about the treatment modalities for each glioma type and grade. Surgery was performed in patients according to the findings of CT scanning, MRI, digital subtraction angiography, and PET. We determined whether postoperative therapy was required according to the histopathological diagnosis. Patients with glioblastoma (Grade IV) and anaplastic astrocytoma (Grade III) were given chemoradiotherapy in accordance with Stupp's regimen after resection. ${ }^{3}$ Patients with astrocytoma (Grade II) underwent resection only without chemoradiotherapy. If the astrocytoma showed progression, we performed surgery and postoperative chemoradiotherapy.
In conclusion, our findings indicate that PRR expression may be thought of as a candidate marker for the prognosis of glioma. As pointed out by Pavlov et al., large-scale prospective studies are needed to fully assess the usefulness of PRR expression as a prognostic marker for glioma.

Masaaki Kouchi, MD, PhD
Yuki Shibayama, PhD
Daisuke Ogawa, MD, PhD
Keisuke Miyake, MD, PhD
Akira Nishiyama, MD, PhD
Takashi Tamiya, MD, PhD
Faculty of Medicine, Kagawa University, Kagawa, Japan

\section{References}

1. Louis DN, Ohgaki H, Wiestler OD, Cavenee WK, Burger PC, Jouvet A, et al: The 2007 WHO Classification of Tumours of the Central Nervous System. Acta Neuropathol 114:97-109, 2007

2. Nguyen G, Muller DN: The biology of the (pro)renin receptor. J Am Soc Nephrol 21:18-23, 2010

3. Stupp R, Mason WP, van den Bent MJ, Weller M, Fisher B, Taphoorn MJ, et al: Radiotherapy plus concomitant and adjuvant temozolomide for glioblastoma. N Engl J Med 352:987-996, 2005

INCLUDE WHEN CITING

Published online July 21, 2017; DOI: 10.3171/2017.2.JNS17254.

(c)AANS, 2017 